\title{
Long non-coding RNA DANCR facilitates glioma malignancy by sponging miR-33a-5p
}

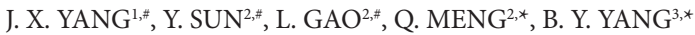 \\ ${ }^{1}$ Department of Neurosurgery, The Second People Hospital of Xin Xiang, Xin Xiang 453003, China; ${ }^{2}$ Department of Neurosurgery, Tangdu \\ Hospital, Fourth Military Medical University, Xian 710038, China; ${ }^{3}$ Department of Neurosurgery, 999 Brain Hospital of Guang Dong, Guang \\ Zhou 510510, China
}

*Correspondence: mengqiang_mq@aliyun.com; baoying2012_yang@21cn.com

${ }^{\#}$ Contributed equally to this work.

Received July 24, 2017 / Accepted November 3, 2017

\begin{abstract}
Glioma is one of the most fatal brain tumors and it is characterized by rapid progression, high malignancy and early metastasis. Deregulation of 'long non-coding RNA differentiation antagonizing non-protein coding RNA' (LncRNA DANCR) is associated with the development, progression and metastasis of various cancers. Herein, we investigated LncRNA DANCR's functional role in glioma malignancy, and results showed that LncRNA DANCR was increased in glioma tissues and cells compared to normal brain tissues and cells, and that its expression positively correlated with the malignancy and poor prognosis of glioma patients. DANCR contains an miR-33a-5p binding site, and miR-33a-5p was also decreased in glioma tissues and cells compared to normal brain tissues and cells. Further, the down-regulation of miR-33a-5p positively correlated with the malignancy and poor prognosis of glioma patients, and DANCR expression in glioma tissue negatively correlated with miR-33a-5p expression. While down-regulation of DANCR increased miR-33a-5p expression, the miR-33a-5p mimic reduced DANCR-WT luciferase but not DANCR-MUT. DANCR pull-down showed the expression of miR-33a-5p, but the miR-33a-5p mimic enhanced the knockdown of DANCR-induced inhibition of cell proliferation, migration and EMT, and increased apoptosis. However, anti-miR-33a-5p reversed the effects of si- DANCR on cell malignancy and DANCR knockdown remarkably reduced the increase of tumor volumes in xenograft mouse models. The knockdown of DANCR increased tumor tissue expression of miR-33a-5p, reduced EMT and increased apoptosis. Our study therefore provides novel insights into the functions of the LncRNA DANCR-miR-33a-5p axis in glioma tumorigenesis.
\end{abstract}

Key words: glioma, LncRNA DANCR, miR-33a-5p, proliferation, invasion, migration

Glioma is one of the most fatal brain tumors and is characterized by high malignancy, rapid progression and early metastasis. While surgical resection combined with radiation therapy and chemotherapy has improved the outcome for glioma patients, they have only median survival of 10-15 months because of its extremely high proliferation and invasiveness [1-4]. The molecular determinants of glioma tumorigenic behavior are not adequately defined, and identification of new biomarkers and molecular target is therefore crucial for the effective treatment of glioma.

Most of the mammalian genome has been confirmed to be noncoding RNAs, together with rapid development in RNA sequencing and high-throughput transcriptome. Long non-coding RNAs (ncRNAs), greater than 200nt in length, are a major type of those noncoding RNAs without protein-coding capability [5]. Although they were originally considered junk RNA with no function, increasing evidence has shown that ncRNAs play essential roles in the regulation of physiological process and pathological conditions, including cancer [6]. Deregulated LncRNAs function similar to oncogenes or tumor suppressors and they have critical roles in different stages of human cancer development; including tumor development, progression, and metastasis [7-10]. Long non-coding RNA differentiation antagonizing non-protein coding RNA (LncRNA DANCR or ANCR) is located on human chromosome 4, near the USP46 and ERVMER34-1 genes.

Studies support LncRNA DANCR's substantial role in the development, progression and metastasis of various cancers. For example, LncRNA DANCR has been reported to be a potential prognostic indicator, promoting cell growth and tumorigenicity in gastric cancer [11], and it also promotes 
invasion of prostate cancer through epigenetically silencing TIMP2/3 expression [12]. While LncRNA DANCR overexpression is associated with advanced tumor progression and poor prognosis in patients with colorectal cancer [13], LncRNA DANCR's role in glioma still remains elusive.

Herein, we designed studies to determine the biological roles of DANCR in glioma. We found that DANCR functions as an oncogenic molecule in glioma and that microRNA miR-33a-5p was the DANCR target that mediated its oncogenic function. These findings indicate that the DANCR/miR-33a-5p interaction is an important regulator in the development and progression of glioma, and they therefore provide new candidates to target glioma treatment.

\section{Materials and methods}

Patients and tissue specimens. The tissue samples, including 10 normal brain tissues and 82 glioma tissues, were obtained from Tangdu Hospital, Fourth Military Medical University between September 2015 and 2016 October 2016. This study was approved by the Research Ethics Committee of Tangdu Hospital, Fourth Military Medical University and informed consent was obtained from all patients. Patients did not receive radiotherapy or chemotherapy before the surgery which removed the tissues, and normal control tissue came from age- and gender-matched patients with cerebral trauma. Tissue samples were kept in liquid nitrogen until the extraction of total RNA. The pathological diagnosis of all glioma patients was performed by two independent experienced clinical pathologists based on WHO classification and patient clinical data including age, gender, WHO grade, type and location was collected. The patients include 34 low grade (grade I-II) and 48 high grade (grade III-IV) individuals and there was a 5 year follow-up in all the patients from the date of surgical resection. The percentage of overall survival (OS) was recorded; with OS defined as the length of time between surgery and death, or the last follow-up examination if death did not occur. The medium cut-off value formed the criteria for patients with high or low levels of lncRNA DANCR and miR-33a-5p.

Bioinformatic data. We used clinical data from the TCGA data portal (https://gdc-portal.nci.nih.gov/) as our other source of samples, and the data of three studies (Glioblastoma (TCGA, Cell 2013); Glioblastoma Multiforme (TCGA, Provisional); Brain Lower Grade Glioma (TCGA, Provisional)) were profiled for expression and survival analysis using cBioportal (http://cbioportal.org).

Cell culture and transfection. The cell lines, including human brain astrocytes (HEB cells) and human glioma cell lines (U87, U251, LN22 9 and T98G) were obtained from the American Type Culture Collection (ATCC, Rockville, MD, USA). The cells were cultured in Dulbecco's Modified Eagle's Medium (DMEM; Gibco, Rockville, MD, USA) supplemented with 10\% fetal bovine serum (FBS; Gibco, Rockville,
$\mathrm{MD}$, USA) and placed in a humidified incubator at $37^{\circ} \mathrm{C}$ with $5 \% \mathrm{CO}_{2}$.

The siNC, siDANCR, miR-33a-5p mimic and inhibitor and their respective negative control RNAs were provided by Ribobio Biotechnology (Guangzhou, China). The si-DANCR, si-NC, miR-33a-5p mimic, NC-mimic, anti-miR-33a-5p and anti-NC were transfected into U87 and LN229 cells with lipofectamine 2000 reagent (Invitrogen, Carlsbad, CA). G418 $(0.5 \mathrm{mg} / \mathrm{ml}$, Sigma-Aldrich, St Louis, MO, USA) was used to establish cell lines with stable DANCR knockdown.

Cell viability. Cell proliferation was performed with Cell Counting Kit-8 (CCK-8; Beyotime, Shanghai, China). Then, $10 \mu \mathrm{L}$ of CCK-8 solution was added to each well after treatment and the cells were incubated at $37^{\circ} \mathrm{C}$ for 2 hours. Absorbance at $450 \mathrm{nM}$ was measured by automatic microplate reader (Synergy 4; BioTek, Winooski, VT, USA). All experimental work was performed in triplicate.

Apoptosis. Apoptotic cell death was measured by TUNELstaining FITC fluorescence kit (Roche, Basel, Switzerland). Cells were then fixed with $4 \%$ paraformaldehyde followed by permeabilization with $0.1 \%$ Triton X-100 and incubated with TUNEL reaction mixture at $37^{\circ} \mathrm{C}$ for 1 hour. Cells were analyzed using FACScan flow cytometer for determination of TUNEL-apoptotic cells (Becton-Dickinson Biosciences, USA).

Invasion and migration assays. Cell invasive ability was measured using a 24-well plate with transwell chamber $(8 \mathrm{~mm}$ pore size, Corning, USA) inserts precoated with Matrigel (BD Biosciences, USA). In brief, $1 \times 10^{5}$ cells were seeded into the upper chamber in $100 \mu \mathrm{l}$ serum-free DMEM medium. The lower chamber was filled with complete medium containing $10 \%$ FBS. After 24 hour incubation, non-migrated cells in the upper surface of the membranes were removed with cotton buds and the invaded cells on the underside of the chamber were fixed with $4 \%$ paraformaldehyde for half an hour and stained with hematoxylin and eosin. The number of invasive cells in six random fields on the bottom surface was counted under a light microscope.

Cells were cultured in a 24-well chamber. After treatment, the confluent cell monolayer was scraped with a pipette tip. Detached and damaged cells were removed by washing and cell migration was monitored by light microscope 24 hours after incubation The migration distance of six preset positions in each group was measured by Image J software.

Quantitative real-time PCR. Total RNA was isolated from cell lines using miRNeasy mini kit (Qiagen, Hilden, Germany). RNA was reversed into cDNA using HighCapacity cDNA Reverse Transcription Kit (Applied Biosystems, Foster City, CA, USA). mRNA expression was quantified by quantitative real-time polymerase chain reaction (PCR) using QuantiTect SYBR Green PCR Kit (Qiagen) on a ABI PRISM 7500 real-time PCR system (Applied Biosystems, Foster City, CA, USA). The amplification was performed by the initial step at $94^{\circ} \mathrm{C}$ for 5 minutes followed by 40 cycles of denaturation at $94^{\circ} \mathrm{C}$ for 30 seconds. Annealing followed 
at $63^{\circ} \mathrm{C}$ for 30 seconds and then extension at $72^{\circ} \mathrm{C}$ for 10 seconds. Data was collected and analyzed by $2^{-\Delta \Delta \mathrm{Ct}}$ method for quantification of relative mRNA expression levels. The target gene values were normalized against GAPDH.

Luciferase reporter assay. The pGL3-DANCR (DANCRWT) was generated by insertion of the DANCR cDNA fragment containing the predicted potential miR-33a-5p binding sites into pGL3-Basic luciferase reporter vector (Promega, Madison, WI, USA). pGL3-DANCR-MUT (DANCR-MUT) was generated by mutation of the potential
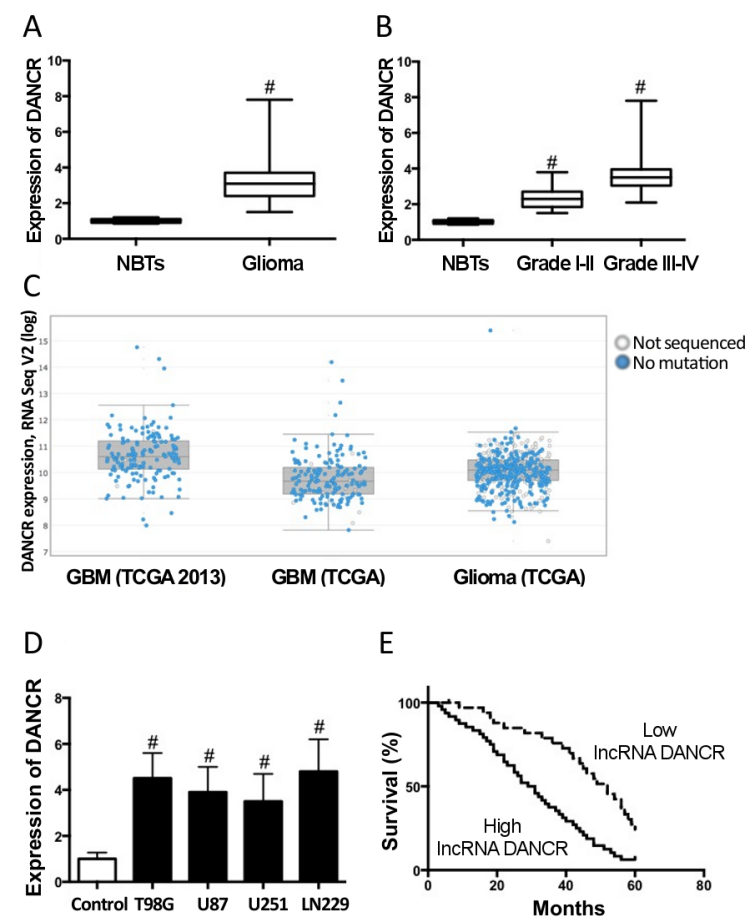

E

F

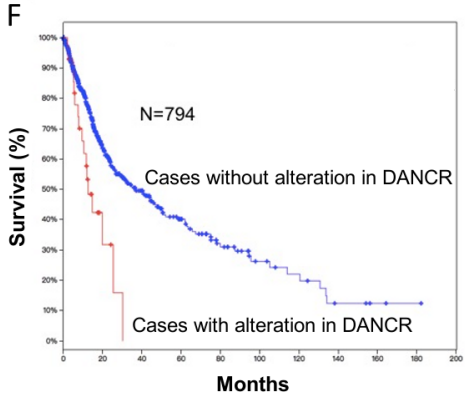

Figure 1. Up-regulation of LncRNA DANCR expression correlates with poor prognosis and negatively correlates with glioma patient miR-33a-5p level. A) LncRNA DANCR expression in 10 normal brain tissues and 82 human glioma tissues was measured by qRT-PCR. B) The LncRNA DANCR expression in low-grade (I-II) and high-grade (III-IV) glioma tissues. C) LncRNA DANCR expression in glioma tissues of patients from the TCGA data set. D) The LncRNA DANCR expression in normal brain and four glioma cell lines determined by $\mathrm{qRT}$-PCR. E) glioma patients were divided into LncRNA DANCR high-expression and low-expression groups and Kaplan-Meier analysis was performed to evaluate the relationship of LncRNA DANCR expression level with prognosis. F) Survival of glioma patients with and without altered LncRNA DANCR expression from the TCGA data set. \#p $<0.05$ compared to control.
miR-33a-5p binding sites, and dual-luciferase reporter assay system performed the luciferase assays (Promega, Madison, WI, USA). Briefly, cells were plated in 96-well plates and co-transfected with $100 \mathrm{ng}$ of the DANCR-WT, or DANCRMUT constructs and $30 \mathrm{ng}$ miR-101-3p mimic or NC-mimic lipofectamine 2000 reagent (Invitrogen, Carlsbad, CA). After 48 hour incubation, the relative luciferase activity was normalized to the Renilla luciferase activity according to manufacturer instructions.

In vivo nude mouse models. Male $\mathrm{BALB} / \mathrm{C}$ nude mice (5-6 weeks of age, 16-18g) were obtained from the Animal Center of Fourth Military Medical University. Animal experiments were approved by the animal experiment committee of Tangdu Hospital, Fourth Military Medical University. Briefly, $4 \times 10^{6}$ U87 cells stably transfected with si-DANCR and si-NC were subcutaneously transplanted into the left flank of the nude mice. Tumor growth in mice was monitored and tumor volumes were calculated at 5-40 days after the transplantation using a vernier caliper. Tumor volume $\left(\mathrm{mm}^{3}\right)=$ length $\times$ width $^{2} / 2$. Mice were then sacrificed and tumor tissues were obtained for further measurement.

Western blot. The total protein samples from tissues were prepared by RIPA lysis buffer (Hycell) containing protease inhibitors (Calbiochem). Total protein was separated on $10 \%$ SDS-PAGE and transferred to PVDF membranes (Millipore, Billerica, USA). Primary antibodies for P-Akt (1:1000; Cell Signaling Technology), Akt (1:1000; Cell Signaling Technology), Cyclin D (1:1000; Cell Signaling Technology), Ki67 (1:1000; Cell Signaling Technology), and $\beta$-actin (1:500; Santa Cruz Biotechnology) were incubated overnight at $4^{\circ} \mathrm{C}$. Membranes were incubated with secondary antibodies conjugated to horseradish peroxidase (Thermo Fisher Scientific, Rockford, IL, USA) for 1 hour at room temperature.

Statistical analysis. Data is recorded as mean \pm SD of three independent experiments and statistical analysis was performed by SPSS 17.0 statistical software (SPSS Inc., Chicago, IL, USA). Student t-test and one-way analysis of variance (ANOVA) were used in either two or multiple groups for statistical significance. Pearson correlation coefficient analyzed correlations between expressions of LncRNA DANCR and miR-33a-5p and the Kaplan-Meier method analyzed the prognostic significance of LncRNA DANCR and miR-33a-5p expression using GraphPad Prism. P-values less than 0.05 were considered statistically significant.

\section{Results}

Expression of LncRNA DANCR indicates poor prognosis of glioma patients. We measured the expression level of LncRNA DANCR in normal brain tissues and glioma tissues. The results showed that the expression level of LncRNA DANCR was increased in glioma tissues compared to normal brain tissue (Figure 1A). The relationship between LncRNA DANCR expression and the degree of pathology in low-grade (I-II) and high-grade (III-IV) glioma tissues was 
examined, and we established that the expression of LncRNA DANCR in high-grade glioma was markedly increased compared to low-grade malignancy (Figure 1B). To further confirm the association of DANCR with glioma risk, we analyzed the gene expression data of glioma patients in the TCGA data set which included three studies (Glioblastoma (TCGA, Cell 2013); Glioblastoma Multiforme (TCGA, Provisional) and found DANCR expression significantly increased (Figure 1C). The expression of LncRNA DANCR in glioma cell lines, including T98G, U87, U251 and LN229 cells, was notably higher than that in human brain astrocytes and HEB cells (Figure 1D). The clinical relevance of DANCR level in glioma was detected by correlation between the expression levels of DANCR and clinical-pathological parameters. These included age, gender, WHO grade, type and location (Table 1). While the expression levels of DANCR were significantly different in WHO grades and increased with tumor grading (Table 1), no significant difference was detected in DANCR expression in age, gender, tumor type or location (Table 1). Figure 1E shows that patients with high LncRNA DANCR expression had shorter OS time than those with low expression $(\mathrm{HR}=1.783,95 \%$ confidence interval $(\mathrm{CI})=1.121-$ 3.4821, $\mathrm{p}=0.0082)$. Data obtained from TCGA showed that patients with altered DANCR had shorter survival time (Figure 1F), and these combined results highlight that LncRNA DANCR indicates poor prognosis in glioma patients.

DANCR is a target of miR-33a-5p. We performed bioinformatics analysis using Starbase v2.0 and found that DANCR has a potential binding site for miR-33a-5p (Figure 2A). DANCR expression was down-regulated in U87 and LN229 cells by siRNAs (Figures 2B, 2C) and this resulted in significant increase in iR-33a-5p expression in these cells (Figures 2D, 2E). The dual-luciferase reporter assay was
A

$\operatorname{miR}-33 a-5 p$

LnCRNA DANCR WT

LncRNA DANCR MUT 5'- CCCCCGCTCCAC-ACCCAACG-3
B

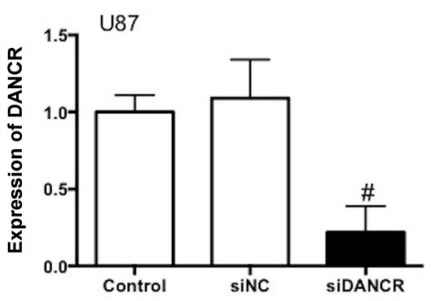

C

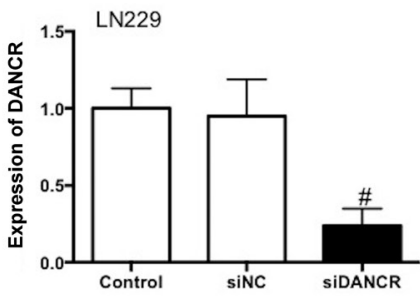

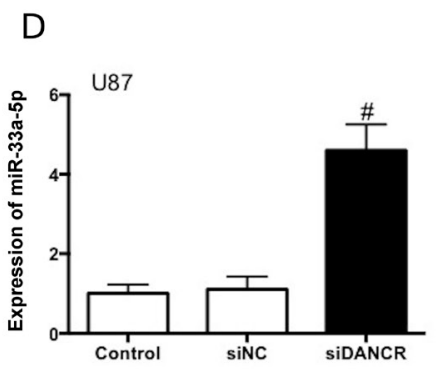
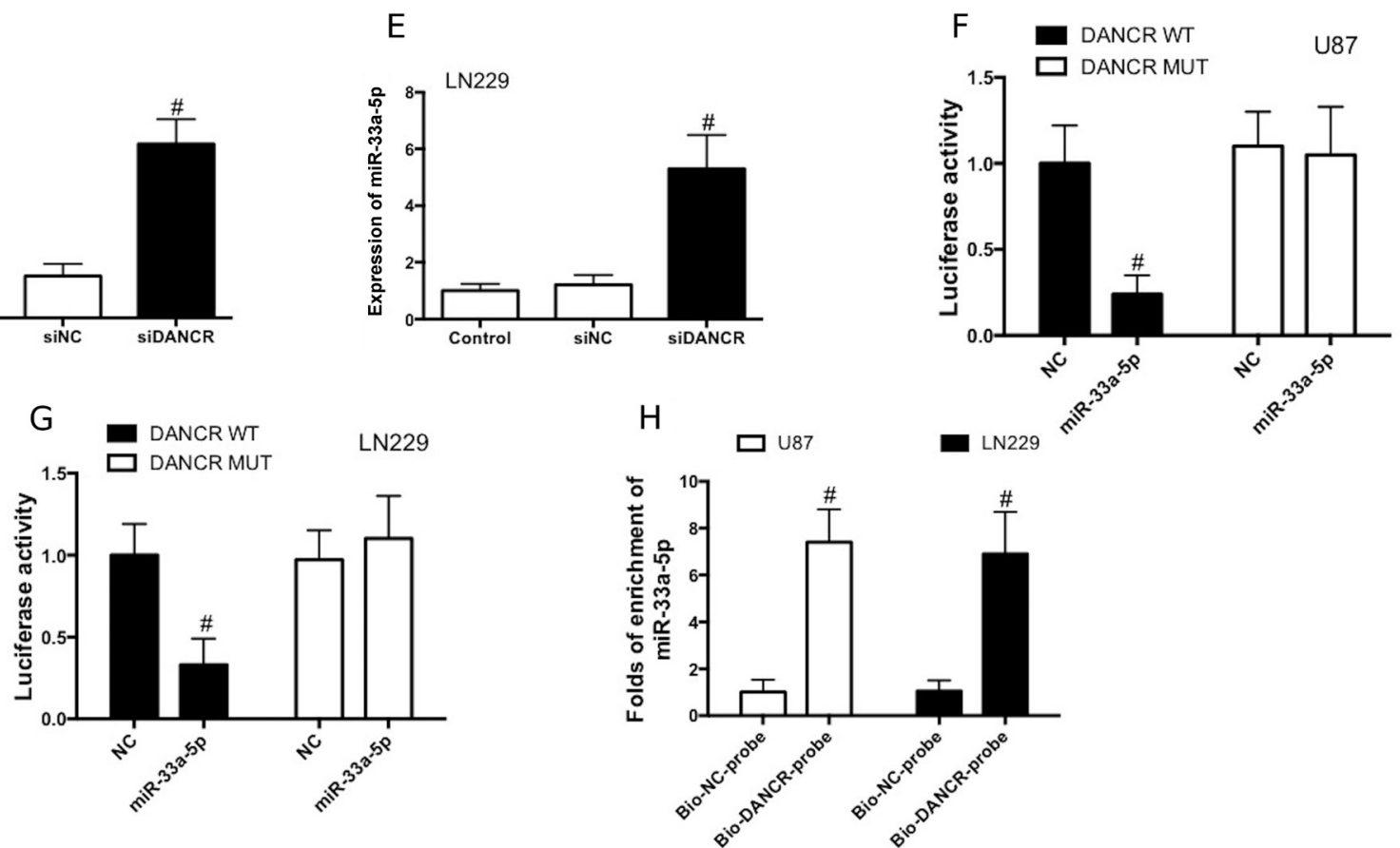

Figure 2. DANCR is a target of miR-33a-5p. A) The predicted binding sites of miR-33a-5p in DANCR (DANCR-WT) and DANCR mutant (DANCRMUT) sequences. B and C) DANCR expression was analyzed by real-time PCR after knockdown of DANCR in U87 and LN229 cells. D and E) miR-33a5p expression was detected in U87 and LN229 cells after si-DANCR transfection. F and G) Luciferase activity was determined in U87 and LN229 cells co-transfected with miR-33a-5p mimic or NC-mimic and pGL3 luciferase reporters containing DANCR-WT or DANCR-MUT sequences. H) Detection of miR-33a-5p using qRT-PCR in the sample pulled down by biotinylated DANCR probe. \#p $<0.05$ compared to control. 

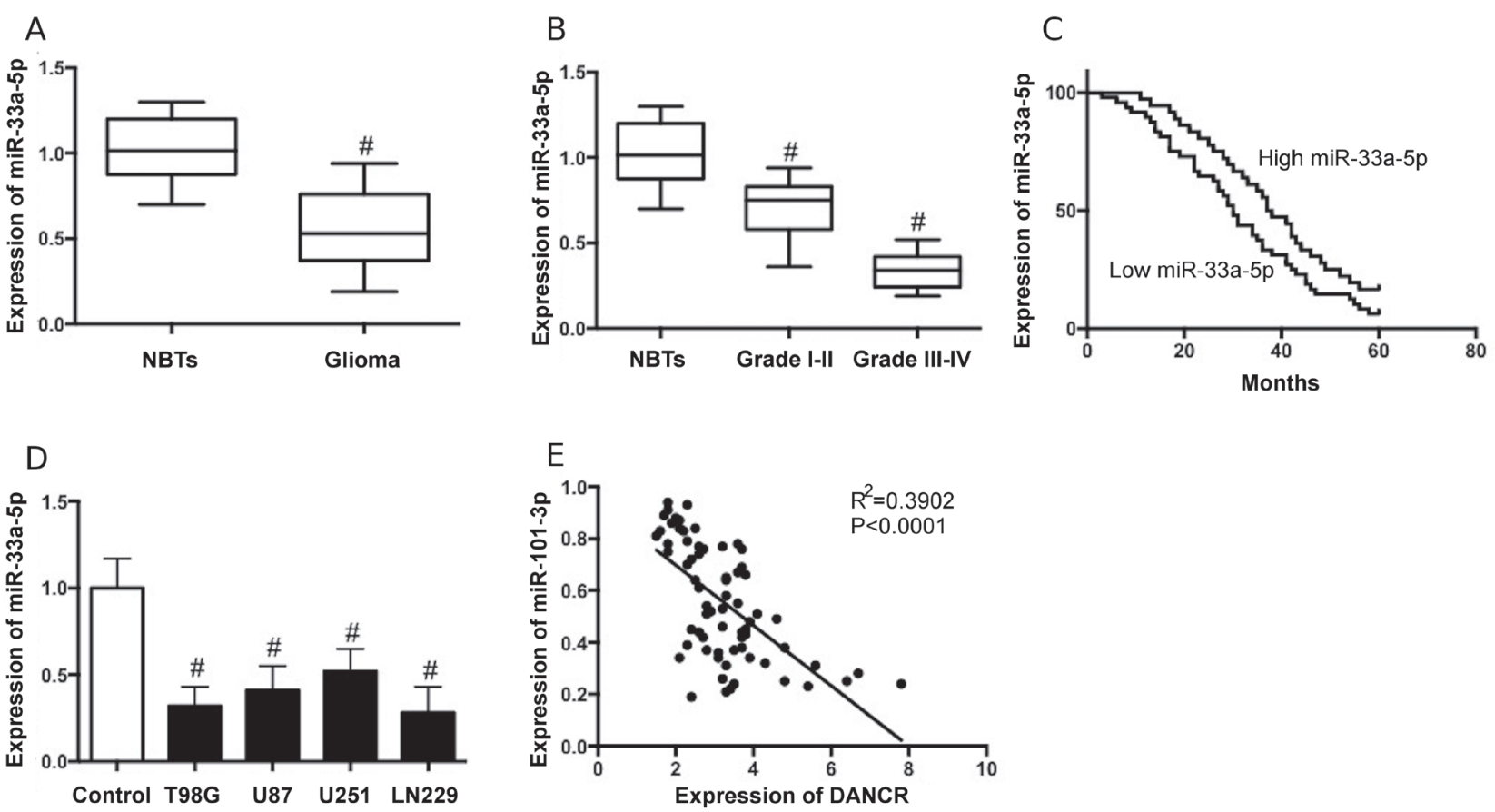

Figure 3. Down-regulation of miR-33a-5p expression correlates with poor prognosis and negatively correlates with glioma patient miR-33a-5p level. A) miR-33a-5p expression in 10 normal brain tissues and 82 human glioma tissues were measured by qRT-PCR. B) miR-33a-5p expression in low-grade (I-II) (29) and high-grade (III-IV) (42) glioma tissues. C) miR-33a-5p expression in normal brain and four glioma cell lines determined by qRT-PCR. D) glioma patients were divided into miR-33a-5p high-expression and low-expression group and Kaplan-Meier analysis evaluated the relationship of miR-33a-5p expression level with prognosis. E) Pearson's correlation analysis of the relationship between LncRNA DANCR and miR-33a-5p. \#p<0.05 compared to control.

conducted to evaluate the regulation of luciferase activity of DANCR by miR-33a-5p mimic. The results showed that miR-33a-5p mimic markedly decreased DANCR-WT (Figures 2F, 2G), whereas miR-33a-5p mimic did not alter the luciferase activity of DANCR-MUT (Figures 2F, 2G). We then performed a pull down assay using biotin-labeled specific DANCR probe, and qRT-PCR revealed that miR-33a-5p was precipitated (Figure $2 \mathrm{H}$ ). The results therefore indicate that DANCR acts as a molecular sponge for miR-33a-5p.

Downregulation of miR-33a-5p indicates poor prognosis of glioma patients. We measured the expression level of miR-33a-5p in normal brain tissues and glioma tissues. The results showed that the expression level of miR-33a-5p was decreased in glioma tissues compared to normal brain tissues (Figure 3A). The relationship between miR-33a-5p expression and the degree of pathology in low-grade (I-II) and high-grade (III-IV) glioma tissues was examined. We showed that the expression of miR-33a-5p in high-grade glioma was markedly reduced compared to low-grade malignancy (Figure 3B). The expression of miR-33a-5p in glioma cell lines, including T98G, U87, U251 and LN229 cells, is notably lower than that in human brain astrocytes and HEB cells (Figure 3C). Figure 3D highlights that patients with high miR-33a-5p expression had longer overall survival time (OS) than those with low miR-33a-5p expression $(\mathrm{HR}=1.426,95 \%$ confidence interval $(\mathrm{CI})=0.973-3.127, \mathrm{p}=0.0105)$. Furthermore, the expression of DANCR and miR-33a-5p in glioma tissues negatively correlate (Figure $3 \mathrm{E}$ ).

DANCR facilitates glioma malignancy through regulation of miR-33a-5p. The U87 and LN229 cells were co-transfected with DANCR and miR-33a-5p or their negative controls in order to evaluate the possible role of DANCR and miR-33a-5p in glioma cell proliferation, invasion, migration and apoptosis. We showed that down- regulation of DANCR markedly decreased cell proliferation (Figures 4A, B), invasion (Figures 4C, D), and migration (Figures 4E, F), and increased E-cadherin expression (Figures 4G, H) and decreased N-cadherin (Figures 4I, J) and Vimentin expression (Figures $4 \mathrm{~K}, \mathrm{~L}$ ).

The miR-33a-5p mimic promoted the effect of DANCR down-regulation on cell proliferation (Figures 4A, B), invasion (Figures 4C, D), and migration (Figures 4E, 4F) and E-cadherin (Figures $4 \mathrm{G}$ and $\mathrm{H}$ ), N-cadherin (Figures 4I, $\mathrm{J})$ and Vimentin expression (Figures $4 \mathrm{~K}, \mathrm{~L}$ ) expression. In contrast, the miR-33a-5p inhibitor significantly suppressed down-regulation of DANCR-induced decrease in cell proliferation (Figures 4A, 4B), invasion (Figures 4C, D), and migration (Figures 4E, F), and the increase in E-cadherin expression (Figures 4G, H) and decrease in $\mathrm{N}$-cadherin (Figures 4I, J) and Vimentin expression (Figures 4K, L). 


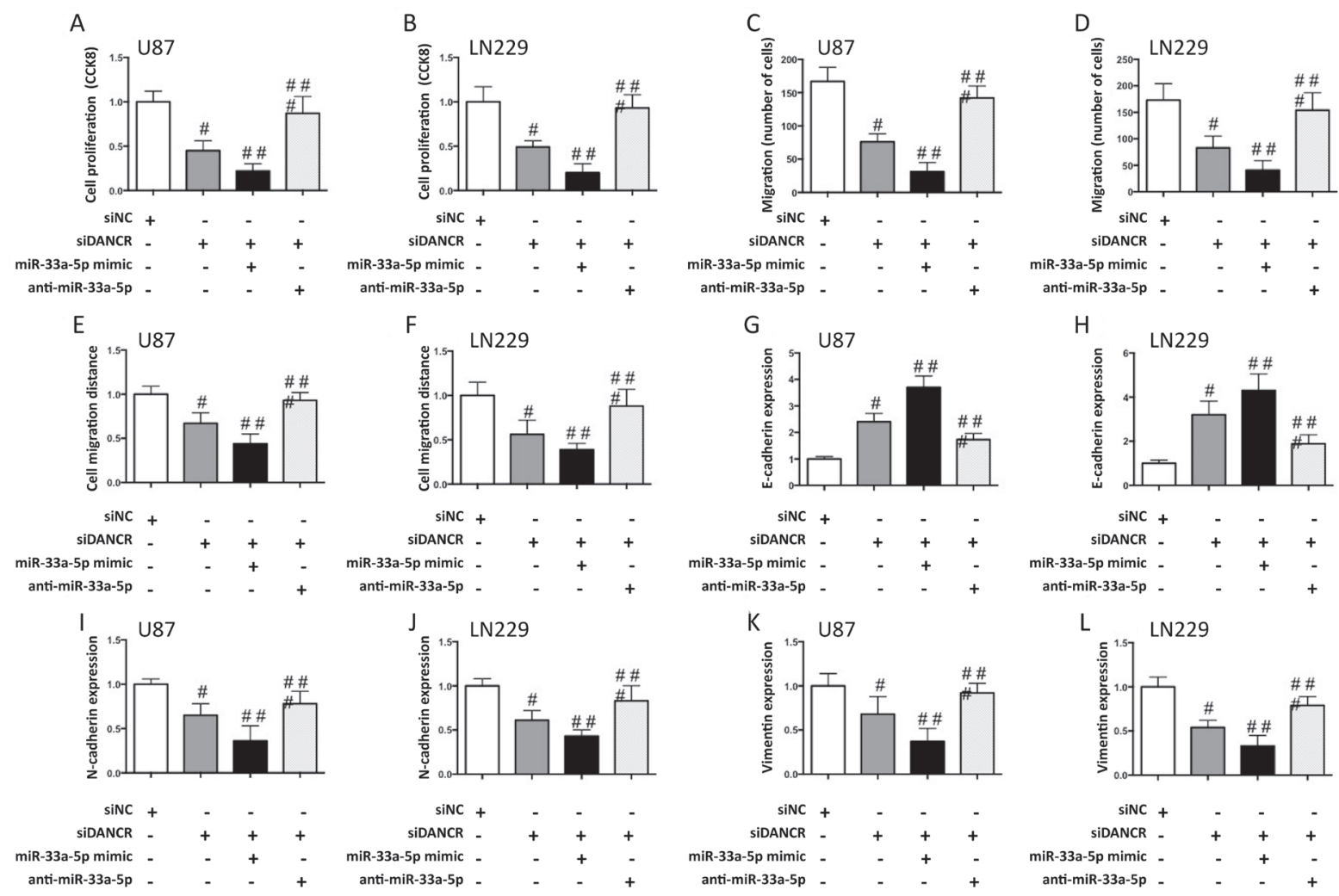

Figure 4. DANCR mediates glioma malignancy by negative regulation of miR-33a-5p. A and B) U87 and LN229 cells was co-transfected with si-DANCR and miR-33a-5p mimic or anti- miR-33a-5p and cell viability was detected by CCK-8 analysis. C and D) The invasion of transfected cells was determined by Transwell assay. E and F) The migration of transfected cells was determined by wounding healing assay; mRNA expression of E-cadherin (G and $\mathrm{H}$ ), N-cadherin ( $\mathrm{I}$ and $\mathrm{J}$ ) and Vimentin ( $\mathrm{K}$ and $\mathrm{L}$ ). \#p $<0.05$ compared to siNC. \#\#p $<0.05$ compared to siDANCR. \#\#\#p $<0.05$ compared to miR-33a-5p.

Moreover, the effect of DANCR and miR-33a-5p on apoptotic cell death in glioma cells was determined. Figures $5 \mathrm{~A}$ and $\mathrm{B}$ show that down-regulation of DANCR induced significant increase in both TUNEL-stained cells and Bax expression (Figures 5C, D) but reduced Bcl-2 expression (Figures 5E, F). While the miR-33a-5p mimic promoted the DANCR down-regulated increase in apoptosis (Figures 5A, B) and expression of Bax (Figures 5C, D) and Bcl-2 (Figures 5E, F), miR-33a-5p significantly suppressed these effects. This indicates that DANCR regulated the glioma cell malignancy by modulating miR-33a-5p expression.

DANCR facilitates glioma tumor growth in xenograft mice. We established xenograft mouse models to evaluate DANCR's role in the regulation of glioma in vivo. Figure 6A shows that DANCR down-regulation resulted in significant inhibition of tumor growth in nude mice. We also measured

Figure 5. DANCR promotes apoptosis by negative regulation of miR-33a5p. A and B) U87 and LN229 cells were co-transfected with si-DANCR and miR-33a-5p mimic or anti- miR-33a-5p, and flow cytometry detected apoptosis. mRNA expression of Bax (C and D) and Bcl-2 (E and F). $\# \mathbf{p}<\mathbf{0 . 0 5}$ compared to siNC. \#\#p $<0.05$ compared to siDANCR. \#\#\#p<0.05 compared to miR-33a-5p.
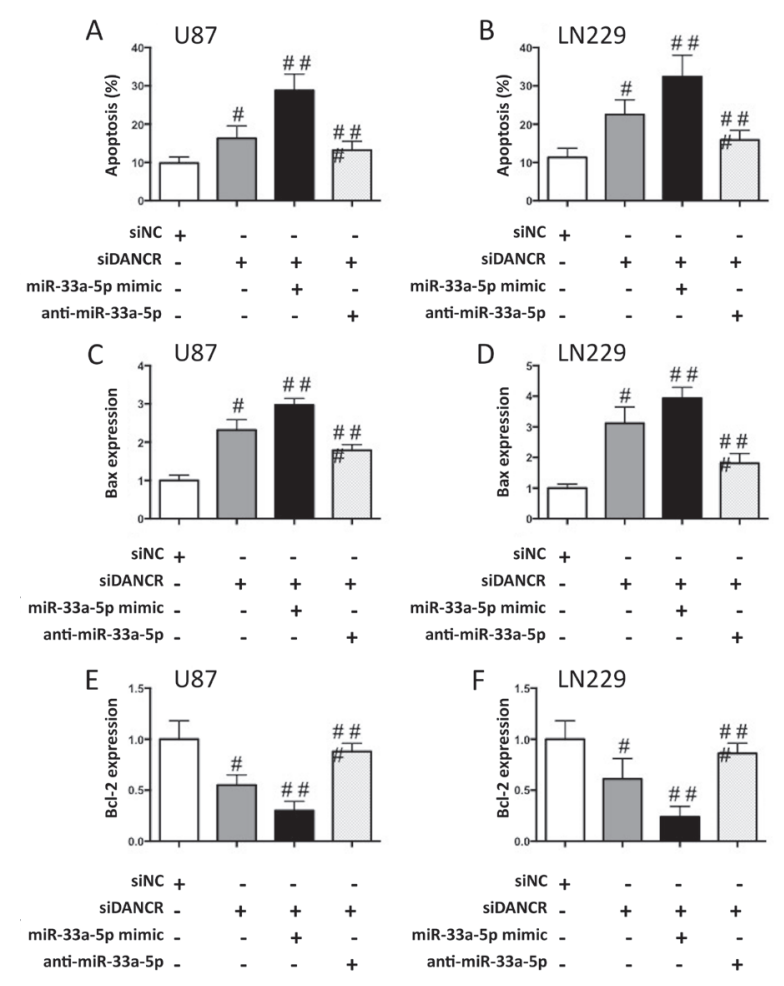
the mRNA expression of EMT and apoptosis biomarkers in tumor tissues, and results confirmed that DANCR downregulation increased miR-33a-5p (Figure 6B) and E-cadherin (Figure 6C) expression, reduced the mRNA expression of $\mathrm{N}$-cadherin (Figure 6D) and Vimentin (Figure 6E), increased Bax expression (Figure 6F) and reduced Bcl-2 expression (Figure 6G). DANCR down-regulation also decreased Akt phosphorylation and expression of Cyclin D and Ki67 in tumor tissues. These findings indicate that DANCR is required for glioma tumor growth in xenograft mice.

\section{Discussion}

LncRNA with transcripts greater than $200 \mathrm{nt}$ in length cannot translate into protein. Emerging evidence has proven that LncRNAs are crucial regulators in various biological processes; and not just transcriptional noise. LncRNAs are believed to be associated with cancer development and progression, metastasis, radio- and chemo-therapy resistance, apoptosis and the epigenetic regulation of tumor biology [7-10]. Increasing studies have provided evidence that LncRNAs serve as biomarkers for early diagnosis, treatment, and prognosis of human cancers. Moreover, LncRNA DANCR plays a substantial role in tumorigenesis because deregulation of LncRNA DANCR is related to pathogenesis in the following cancers; gastric [11], prostate [12], colorectal [13], osteosarcoma [14] and hepatocellular carcinoma $[15,16]$.

Herein, we tested the role of LncRNA DANCR in the development and progression of glioma and clarified the possible mechanism involved. We found that LncRNA DANCR played an oncogenic role in the development of glioma and promoted malignant transition. The LncRNA DANCR expression level in glioma tissue is higher than that
A

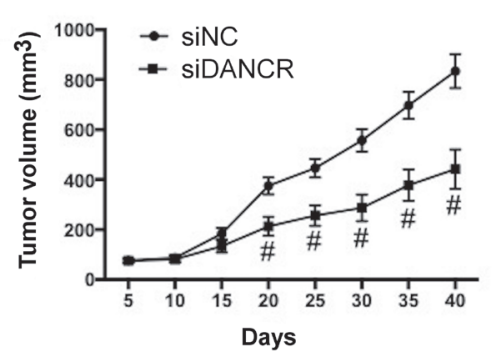

D

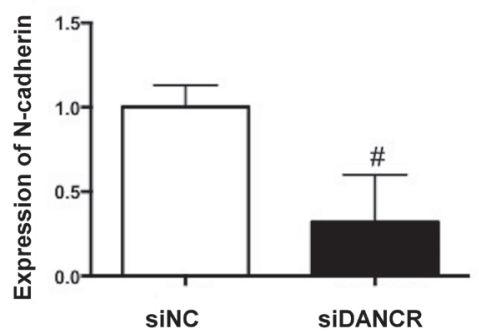

G

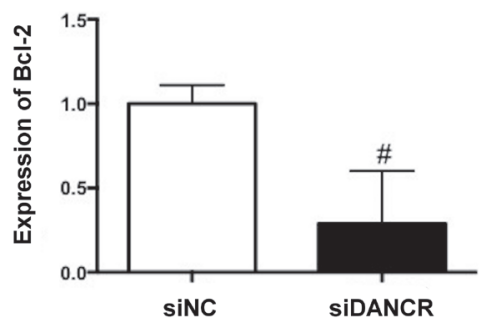

B

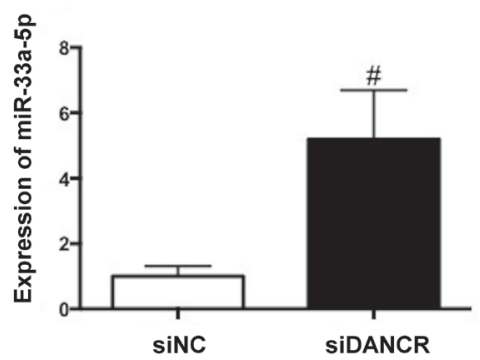

E

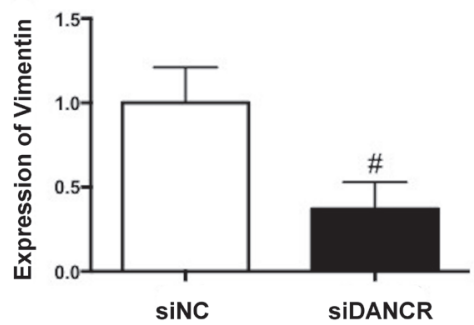

$\mathrm{H}$

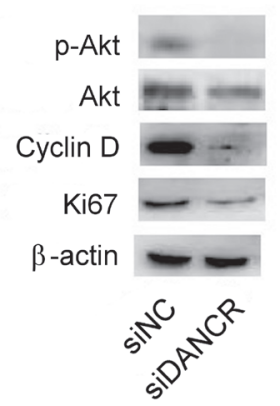

C

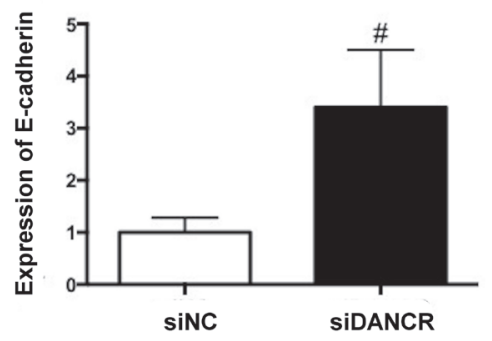

$\mathrm{F}$

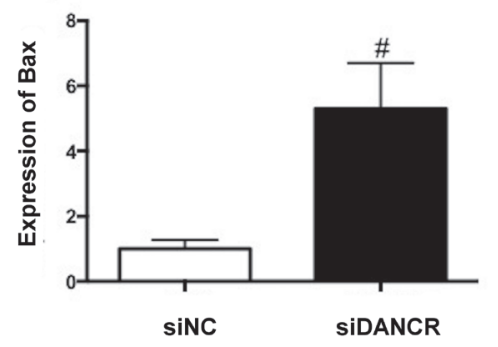

।

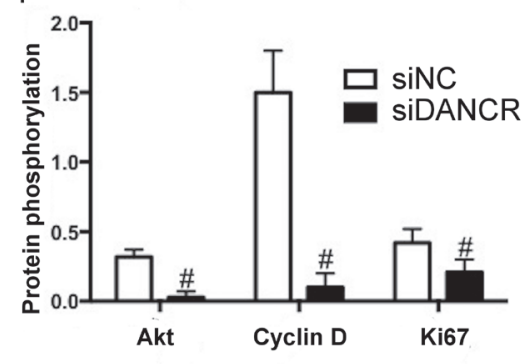

Figure 6. SNHG6 promotes glioma tumor growth in the xenograft mouse model. A) The U87 cells stably transfected with si-DANCR and si-NC subcutaneously injected into nude mice and tumor volumes were measured. mRNA expression of miR-33a-5p (B), E-cadherin (C), N-cadherin (D), Vimentin (E), Bax (F), and Bcl-2 (G). H and I) Protein expression of phosphorylated Akt, Akt, Cyclin D and Ki67 and statistical analysis of protein bands. $\# \mathrm{p}<0.05$ compared to siNC. 
in normal brain tissue and it increases in line with glioma malignant transition. Moreover, TCGA confirmed that patients with high LncRNA DANCR expression had shorter OS time than those with low expression and its down-regulation inhibited cell proliferation in vitro and tumor growth in vivo. The LncRNA DANCR down-regulation also decreased tumor invasion and migration ability, increased apoptosis and induced abnormal expression of EMT biomarkers in glioma cells and tumor tissues. These combined results suggest that LncRNA DANCR has an oncogenic role in glioma malignancy and that it could serve as a prognostic marker for glioma patients.

While exploring mechanisms which mediate LncRNA DANCR oncogenic function in glioma, we found that miR-33a-5p was a LncRNA DANCR target, and we identified miR-33a-5p's potential binding site in the DANCR sequence. While DANCR down-regulation increased miR-33a-5p expression in vitro and in vivo, the miR-33a-5p mimic reduced luciferase activity of DANCR-WT but not DANCRMUT. Pull-down of DANCR confirmed the direct interaction between DANCR and miR-33a-5p. Further, we showed that the expression level of miR-33a-5p in glioma tissues was lower than in normal brain tissues, that it was reduced in tandem with glioma malignant transition and that patients with high miR-33a-5p expression had longer OS time than those with low expression.

DANCR and miR-33a-5p expression negatively correlate in clinical glioma tissues. DANCR has a binding site for miR-33a-5p and IP results confirmed this binding. This indicates that DANCR can act as a competing endogenous RNA for miR-33a-5p and regulate downstream targets.

Authors report that microRNAs regulate target gene expression by inducing mRNA degradation or suppressing mRNA translation through base-pairing with the $3^{\prime}$-UTR of the mRNA $[17,18]$, and also highlight that microRNA has a key role in mediating LncRNA biological functions; including the regulation of tumor development [19-22]. Studies have shown the following results of miR-33a-5p deregulation; (1) miR-33a-5p increased radio-sensitivity in melanoma by inhibiting glycolysis in melanoma [23]; (2) down-regulated miR-33a-5p is a possible mechanism for chemotherapy resistance in hepatocellular carcinoma [24]; (3) miR-33a-5p modulates TNF-alpha-inhibited osteogenic differentiation by targeting SATB2 expression in hBMSCs [25]; (4) miR-33a-5p is down-regulated in human osteosarcoma and suppresses cell growth [26] and (5) most importantly, miR-33a-5p is identified as the LncRNA DANCR target in regulation of tumor progression and cancer-stem features in osteosarcoma [14]. All these results indicate that the LncRNA DANCR-miR-33a-5p axis is a common pathway contributing to tumorigenesis in different cancers.

In conclusion, DANCR expression is increased in glioma tissues and cells. It facilitates cancer cell proliferation, invasion and metastasis and inhibits apoptosis by competitive binding to miR-33a-5p. The LncRNA DANCR-miR- 101-3p axis appears a key regulator of glioma metabolism and progression, and further investigation of this confirmed regulatory network could well provide a novel target for glioma therapy.

\section{References}

[1] SIEGEL RL, MILLER KD, JEMAL A. Cancer statistics, 2016. CA Cancer J Clin 2016; 66: 7-30. https://doi.org/10.3322/ caac. 21332

[2] SHERMAN JH1, HOES K, MARCUS J, KOMOTAR RJ, BRENNAN CW et al. Neurosurgery for brain tumors: update on recent technical advances. Curr Neurol Neurosci Rep 2011; 11: 313-319. https://doi.org/10.1007/s11910-0110188-9

[3] WEN PY, KESARI S. Malignant gliomas in adults. N Engl J Med 2008; 359: 492-507. https://doi.org/10.1056/NEJMra0708126

[4] SATHORNSUMETEE S, RICH JN. New treatment strategies for malignant gliomas. Expert Rev Anticancer Ther 2006; 6: 1087-1104. https://doi.org/10.1586/14737140.6.7.1087

[5] KHALIL AM, GUTTMAN M, HUARTE M, GARBER M, RAJ A et al. Many human large intergenic noncoding RNAs associate with chromatin-modifying complexes and affect gene expression. Proc Natl Acad Sci U S A 2009; 106: $11667-$ 11672. https://doi.org/10.1073/pnas.0904715106

[6] PONTING CP, OLIVER PL, REIK W. Evolution and functions of long noncoding RNAs. Cell 2009; 136: 629-641. https://doi.org/10.1016/j.cell.2009.02.006

[7] LIU B, SUN L, LIU Q, GONG C, YAO Y et al. A cytoplasmic NF-kappaB interacting long noncoding RNA blocks IkappaB phosphorylation and suppresses breast cancer metastasis. Cancer Cell 2015; 27: 370-381. https://doi.org/10.1016/j. ccell.2015.02.004

[8] LI T, XIE J, SHEN C, CHENG D, SHI Y et al. Amplification of Long Noncoding RNA ZFAS1 Promotes Metastasis in Hepatocellular Carcinoma. Cancer Res 2015; 75: 3181-3191. https://doi.org/10.1158/0008-5472.CAN-14-3721

[9] POLISENO L, SALMENA L, ZHANG J, CARVER B, HAVEMAN WJ et al. A coding-independent function of gene and pseudogene mRNAs regulates tumour biology. Nature 2010; 465: 1033-1038. https://doi.org/10.1038/nature09144

[10] MATTICK JS, MAKUNIN IV. Non-coding RNA. Hum Mol Genet 2006; 1: R17-29. https://doi.org/10.1093/hmg/ddl046

[11] HAO YP, QIU JH, ZHANG DB, YU CG. Long noncoding RNA DANCR, a prognostic indicator, promotes cell growth and tumorigenicity in gastric cancer. Tumour Biol 2017; 39: 1010428317699798. https:/doi. org/10.1177/1010428317699798

[12] JIA J, LIF, TANG XS, XU S, GAO Y et al. Long noncoding RNA DANCR promotes invasion of prostate cancer through epigenetically silencing expression of TIMP2/3. Oncotarget 2016; 7: 37868-37881. https://doi.org/10.18632/oncotarget.9350

[13] LIU Y, ZHANG M, LIANG L, LI J, CHEN YX. Over-expression of lncRNA DANCR is associated with advanced tumor progression and poor prognosis in patients with colorectal cancer. Int J Clin Exp Pathol 2015; 8: 11480-11484. 
[14] JIANG N, WANG X, XIE X, LIAO Y, LIU N et al. lncRNA DANCR promotes tumor progression and cancer stemness features in osteosarcoma by upregulating AXL via miR-33a5p inhibition. Cancer Lett 2017; 405: 46-55. https://doi. org/10.1016/j.canlet.2017.06.009

[15] MA X, WANG X, YANG C, WANG Z, HAN B et al. DANCR Acts as a Diagnostic Biomarker and Promotes Tumor Growth and Metastasis in Hepatocellular Carcinoma. Anticancer Res 2016; 36: 6389-6398. https://doi.org/10.21873/ anticanres. 11236

[16] YUAN SX, WANG J, YANG F, TAO QF, ZHANG J et al. Long noncoding RNA DANCR increases stemness features of hepatocellular carcinoma by derepression of CTNNB1. Hepatology 2016; 63: 499-511. https://doi.org/10.1002/ hep. 27893

[17] RIVERA-BARAHONA A, PEREZ B1, RICHARD E1, DESVIAT LR. Role of miRNAs in human disease and inborn errors of metabolism. J Inherit Metab Dis 2017; 40: 471-480. https://doi.org/10.1007/s10545-017-0018-6

[18] WANG ZH, XU CJ. Research Progress of MicroRNA in Early Detection of Ovarian Cancer. Chin Med J (Engl) 2015; 128: 3363-3370. https://doi.org/10.4103/0366-6999.171459

[19] DU Y, LI J, XU T, ZHOU DD, ZHANG L et al. MicroRNA-145 induces apoptosis of glioma cells by targeting BNIP3 and Notch signaling. Oncotarget 2017; 8: 61510-61527. https://doi.org/10.18632/oncotarget.18604

[20] LI B, LIU YH, SUN AG, HUAN LC, LI HD et al. MiR-130b functions as a tumor promoter in glioma via regulation of ERK/MAPK pathway. Eur Rev Med Pharmacol Sci 2017; 21: 2840-2846.
[21] LAN T, MA W, HONG Z, WU L, CHEN X et al. Long noncoding RNA small nucleolar RNA host gene 12 (SNHG12) promotes tumorigenesis and metastasis by targeting miR199a/b-5p in hepatocellular carcinoma. J Exp Clin Cancer Res 2017; 36: 11. https://doi.org/10.1186/s13046-016-0486-9

[22] LIU J, LU C, XIAO M, JIANG F, QU L et al. Long non-coding RNA SNHG20 predicts a poor prognosis for HCC and promotes cell invasion by regulating the epithelial-to-mesenchymal transition. Biomed Pharmacother 2017; 89: 857-863. https://doi.org/10.1016/j.biopha.2017.01.011

[23] CAO K, LI J, CHEN J, QIAN L, WANG A et al. microRNA$33 a-5 p$ increases radiosensitivity by inhibiting glycolysis in melanoma. Oncotarget 2017; 8: 83660-83672. https://doi. org/10.18632/oncotarget.19014

[24] MENG W, TAI Y, ZHAO H, FU B, ZHANG T et al. Downregulation of miR-33a-5p in Hepatocellular Carcinoma: A Possible Mechanism for Chemotherapy Resistance. Med Sci Monit 2017; 23: 1295-1304.

[25] MI W, SHI Q, CHEN X, WU T et al. miR-33a-5p modulates TNF-alpha-inhibited osteogenic differentiation by targeting SATB2 expression in hBMSCs. FEBS Lett 2016; 590: 396407. https://doi.org/10.1002/1873-3468.12064

[26] ZHANG J, WANG D, XIONG J, CHEN L, HUANG J. MicroRNA-33a-5p suppresses growth of osteosarcoma cells and is downregulated in human osteosarcoma. Oncol Lett 2015; 10: 2135-2141. https://doi.org/10.3892/ol.2015.3503 